\title{
Síndrome de Burnout: Un estudio con docentes de Educación Básica
}

\section{Burnout syndrome: A study with teachers of Basic Education}

\author{
Patricia Katherine Vega-Feijoó \\ patricia.vega@psg.ucacue.edu.ec \\ Universidad Católica de Cuenca, Cuenca \\ Ecuador \\ https://orcid.org/0000-0001-5485-9775 \\ Darwin Gabriel Garcia-Herrera \\ dggarciah@ucacue.edu.ec \\ Universidad Católica de Cuenca, Azogues \\ Ecuador \\ https://orcid.org/0000-0001-6813-8100 \\ Sergio Constantino Ochoa-Encalada \\ scochoae@ucacue.edu.ec \\ Universidad Católica de Cuenca, Azogues \\ Ecuador \\ https://orcid.org/0000-0003-3067-3719 \\ Juan Carlos Erazo-Álvarez \\ jcerazo@ucacue.edu.ec \\ Universidad Católica de Cuenca, Cuenca \\ Ecuador \\ https://orcid.org/0000-0001-6480-2270
}

Recibido: 26 de abril de 2020

Revisado: 15 de mayo de 2020

Aprobado: 10 de junio de 2020

Publicado: 29 de junio de 2020 


\title{
RESUMEN
}

La presente investigación tuvo como objetivo analizar el Síndrome de Burnout (estrés laboral) en docentes de Educación Básica de diferentes Unidades Educativas de la ciudad de Cuenca-Ecuador, el estudio se desarrolló a través de un enfoque cuantitativo, no experimental y cohorte transversal, los datos se recolectaron a través de la aplicación del Maslach Burnout Inventory (MBI), el análisis estadístico de la información, revela un agotamiento emocional en el $28,57 \%$ de los docentes, despersonalización en el $17.14 \%$ y una baja realización personal en el $54,28 \%$; resultados que evidenciaron índices bajos de estrés laboral, pero sin embargo muy cercanos a los umbral mínimos, dejando entrever la posibilidad de trabajar enfáticamente en la realización personal y la satisfacción laboral, como alternativas para combatir los estresores que agotan la práctica docente. La propuesta a implementar para mejorar los niveles de estrés laboral, se enfocaría en la implementación de talleres y pausas activas que permitan atender tanto la parte emocional como física del docente.

Descriptores Estrés mental; enfermedad profesional; voluntad de realización; docente. (Palabras tomadas del Tesauro UNESCO).

\begin{abstract}
The objective of this research was to analyze Burnout Syndrome (work stress) in Basic Education teachers from different Educational Units of the city of Cuenca-Ecuador, the study was developed through a quantitative, non-experimental and cross-sectional cohort approach, The data was collected through the application of the Maslach Burnout Inventory $(\mathrm{MBI})$, the statistical analysis of the information, reveals emotional exhaustion in $28.57 \%$ of the teachers, depersonalization in $17.14 \%$ and low personal achievement in the 54.28\%; results that evidenced low rates of work stress, but nevertheless very close to the minimum thresholds, hinting at the possibility of emphatically working on personal achievement and job satisfaction, as alternatives to combat stressors that exhaust teaching practice. The proposal to implement to improve levels of work stress, would focus on the implementation of workshops and active breaks that allow attending both the emotional and physical part of the teacher.
\end{abstract}

Descriptors: Mental stress; occupational diseases; achievement motivation; teachers. (Words taken from the UNESCO Thesaurus). 


\section{INTRODUCCIÓN}

La sociedad actual en su frenético crecimiento exige el incremento de mayores habilidades por parte de los trabajadores, (Carlotto \& Câmara, 2017), (Quintero, Vergara, Hernández, Osorio, \& Orozco, 2018) y (Arias, Huamani, \& Ceballos, 2019), manifestan que profesiones como la docencia que mantiene relación directa con otras personas, suponen una relación laboral de alto riego. Conflictos permanentes entre pares, baja motivación del estudiantado, la exigencia de padres, escases de recursos, grupos numerosos y el poco incentivo, dan como resultado diversas manifestaciones emocionales y físicas, mismas que están generando niveles elevados de estrés laboral en los docentes.

A decir de (Silva, García, González, \& Ratto, 2015), (Rabasa, Rabasa, Gil-Monte, \& Llorca-Pellicer, 2016), el denominado estrés laboral se ha posicionado como una de las enfermedades de mayor crecimiento en los últimos años, según la investigación realizada por (González, 2008), refiere que alrededor de un $60 \%$ de trabajadores que evidencian ausentismo laboral lo hacen como consecuencia de la excesiva carga laboral. (Contreras \& Burguillos, 2014), describe que el estrés relacionado con el trabajo ha supuesto, según estudios realizados por Unión Europea, el segundo problema más común.

En palabras de (Zavala, 2008), las afectaciones que pueden tener los docentes son muy variadas, éstas pueden disminuir y perturbar tanto su parte física como psicológica afectando de manera significativa tanto su relación laboral como familiar. Algunos de los síntomas que en mayor proporción se presentan son: dificultad para concertarse, insomnio, angustia, ansiedad, gastritis, dolor de espalda, cuello y cabeza, espasmos musculares, tartamudeo, debilidad, vértigo, entre otras.

La palabra "estrés", ampliamente estudiada, proviene del francés "stress" cuyo significado supone estar bajo cierta presión o estreches, más adelante al surgir de un inglés medieval fue tomado como "distrés", varios autores señalan que todas las actividades que el ser humano desarrolla generan respuestas adaptativas, generando un grado de estrés que puede ser positivo o negativo, todo depende de la respuesta diversa que como individuos podamos otorgar, sin embargo si ésta es negativa y se 
alarga en el tiempo puede ocasionar el denominado "Síndrome de Burnout" (Bambula \& Gómez, 2016).

Con base en las exploraciones anteriores, la presente investigación tiene como objetivo analizar Síndrome de Burnout en docentes, mediante la manifestación de sus tres dimensiones: agotamiento emocional, despersonalización y la realización personal, especialmente durante estos días de incertidumbre que atraviesa el país, fundamentalmente los docentes, como consecuencia de la pandemia declarada a nivel mundial.

\section{Referencial teórico}

Estudios en América Latina, refieren que las condiciones de los Sistemas Educativos poseen factores que inciden de manera negativa en la prevalencia del estrés asociado a docentes, Ecuador no es la excepción, un estudio realizado en la Universidad de Autónoma de los Andes con una muestra 52 docentes de los cuales 25 son mujeres $(44,6 \%)$ y 31 son hombres (55,3\%), reveló que el $12,6 \%$ sufren o padecen Síndrome de Burnout. Además, se pudo evidenciar la existencia diversos factores de riegos, que no deben ser tomados a la ligera (Romero, Pimienta, Ramos, Sánchez, \& González, 2019).

Desde una perspectiva nacional, un estudio realizado por (Rivera, Segarra, \& Valverde, 2018), en la ciudad de Guayaquil, reveló en sus conclusiones que docentes universitarios que llevaban más de una década al servicio educativo presentaban síntomas asociados al burnout, si bien no existió un diagnóstico general en la totalidad de su muestra, conformada por 270 docentes, si se evidencia una tendencia clara hacia el agotamiento, cansancio, poca satisfacción, baja autoestima, entre otras, signos alarmantes a desencadenar en un síndrome del quemado. Para aquellos que ya poseen afectación o sintomatología se debería implementar estrategias que contribuyan a mejorar esta situación y no favorezcan su prolongación mermando la salud y capacidad de sus docentes. 


\section{El estrés laboral}

Para conocer más a profundidad el termino burnout, es imperativo citar al psicólogo estadounidense Herbert Freudenberg, quien en los años setenta observó manifestaciones psíquicas y físicas de un grupo específico de voluntarios que debido al trabajo intenso generó agotamiento, a tal punto de no tomar en cuenta sus propias necesidades y quemarse. Más adelante, en 1981 Cristina Maslach, acuñó dicha manifestación con el término de "Burnout", de hecho, se diferencian cuatro etapas: entusiasmo, estancamiento, frustración y burnout propiamente dicho (Gallegos \& Barrios, 2013); (Contreras \& Burguillos, 2014); (Villaverde, Unda, Escotto, \& Flores, 2019).

Para (Zavala, 2008), "El burnout también puede interpretarse como una capacidad de respuesta no adecuada ante una diversidad de demandas no satisfactorias" (p. 71). Mientras que para (Rivera, Segarra, \& Valverde, 2018), el estrés que sufren los docentes viene acompañado de la excesiva carga documental que ocasiona una disminución en su estado de armonía teniendo un impacto negativo en su salud y por ende en su forma de enseñar. En palabras de (Contreras \& Burguillos, 2014), el Síndrome de Burnout causa secuelas graves a nivel físico y psicológico presentándose como una patología severa que se relaciona directamente con el trabajo y estilo de vida.

\section{Dimensiones del estrés laboral}

En palabras de (Arias, Sánchez, \& Ceballos, 2017) y (Rivera, Segarra, \& Valverde, 2018), el síndrome de Burnout se manifiesta con numerosos síntomas afectando directamente tres áreas: agotamiento emocional, despersonalización y realización personal.

Agotamiento Emocional se exterioriza con una creciente desmotivación en su trabajo, desinterés, profundo abatimiento que genera en él su incapacidad para dar más de sí mismo.

Despersonalización genera en la persona un estado de aislamiento pues siente que así está protegido, posee una actitud negativa y trato distante a los estudiantes y a quien lo rodea. 
Baja realización personal la persona siente que no puede superarse y ello afecta su autoestima y autorrealización, prefiere retirarse para no seguir sufriendo.

El estrés severo al presentarse como una patología que disminuye la capacidad del docente tanto en su parte física como emocional precisa de estrategias que puedan ser implementadas de manera eficaz, para ello es imperativo tomar en cuenta los resultados de esta investigación y formular una propuesta que atienda las dimensiones que se vean más afectadas.

\section{MÉTODO}

La presente investigación obedece a un enfoque cuantitativo, es de carácter no experimental y de corte transversal puesto que se recolectó los datos en un solo momento (Vintimilla-Veloz, Erazo-Álvarez, \& Narváez-Zurita, 2020), la muestra se estableció a través de una selección aleatoria simple y estuvo constituida por 350 docentes de diferentes Unidades Educativas de la ciudad de Cuenca, el 85,71\% son mujeres y el $14,28 \%$ son varones, sus edades oscilaban entre los 20 y 60 años.

EL instrumento aplicado para recolectar los datos consta de dos partes: la primera que recoge datos demográficos como la edad, género, experiencia laboral y nivel de instrucción y la segunda que consta del test de Maslach Burnout Inventory conocido por su gran prestigio en investigaciones psicológicas y educativas, está conformado por una escala tipo Likert que posee 22 ítems que van de 0 (nunca) a 6 (todos los días).

El instrumento se rige por tres parámetros (ver tabla 1): agotamiento emocional (1, 2, 3, 6, 8, 13, 14, 16,20 ítems), despersonalización (5,10, 11, 15, 22 ítems) y realización personal $(4,7,9,12,17,19,21$ ítems), para el presente estudio se validó el instrumento obteniendo .703 según el alfa de Cronbach (Esteras, Chorot, \& Sandín, 2019). 


\section{Tabla 1}

Puntuaciones según el Test de Maslach

\begin{tabular}{cccc} 
Aspecto evaluado & Bajo & Medio & Alto \\
\hline Agotamiento & $0-18$ & $19-26$ & $27-54$ \\
emocional & $0-5$ & $6-9$ & $10-30$ \\
Despersonalización & $0-33$ & $34-39$ & $40-56$ \\
Realización personal & &
\end{tabular}

Fuente: (Arias, Sánchez, y Ceballos, 2017).

Para la recolección de datos se transformó el instrumento delimitado a un formulario de Google Form, para posteriormente compartirlo mediante Whatsapp y su aplicación de forma anónima asegurando su plena confidencialidad. Una vez recogidos los datos, se procedió a validar el instrumento mediante análisis de confiabilidad para luego realizar un análisis estadístico descriptivo a través de Statistical Software Package (SPSS).

\section{RESULTADOS}

Para el análisis de los datos se realizó una comparación entre las variables correspondientes a las 3 dimensiones (Agotamiento emocional, despersonalización y Baja realización personal) que integra el test de Maslach y también se cruzaron los datos en función de las variables demográficas.

Al calcularse los datos estadísticos descriptivos se revela que los puntajes de la media en cuanto a la realización personal se encuentran en los niveles superiores, demostrando estabilidad en el plano laboral, además se evidencia que el puntaje de la media para la Despersonalización y el Agotamiento emocional son negativos, dejando entrever escasos niveles del Síndrome de Burnout en los profesores, sin embargo es necesario considerar que los valores establecidos están próximos al umbral para el establecimiento de Síndrome de Burnout y por ende muestran una tendencia a incrementar, en caso de no tomarse las medidas preventivas adecuadas. 


\section{Tabla 2}

Número de docentes en los niveles del síndrome de Burnout

\begin{tabular}{lccc} 
Aspecto evaluado & Bajo\% & Medio\% & Alto\% \\
\hline Agotamiento emocional & 57,14 & 14,28 & 28,57 \\
Despersonalización & 57,14 & 25,71 & 17,14 \\
Realización personal & 20,00 & 25,71 & 54,28 \\
\hline
\end{tabular}

Fuente: Elaboración propia

La Tabla 2 revela que, del total de docentes encuestados, en los niveles altos del Síndrome de Burnout se encuentran en el 28,57\% y están asociados al agotamiento emocional y un 17,14\% a la despersonalización, por el contrario, la realización en el trabajo se posiciona con niveles altos siendo $54.28 \%$. Estos indicadores evidencian que, si bien no hay un marcado Síndrome de Burnout, los docentes presentaron en alguna ocasión o presentan situaciones tales como: una baja autoestima, culpabilidad, poca relación con los estudiantes, sentimientos de inferioridad e irritabilidad y se sienten poco valorados en sus funciones.

Prevalece estrés alto en casi una tercera parte de los docentes, de continuar la presión podría llegarse a un punto de inflexión que impactaría en su aspecto físico y psicológico y desencadenaría mayores índices a los mencionados. Interpretándose así que estos profesores son un factor de riesgo hacia un estrés crónico o Síndrome de Burnout.

\section{Tabla 3}

Estadística descriptiva de las dimensiones del Burnout

\begin{tabular}{|c|c|c|c|c|c|c|}
\hline & $\mathbf{N}$ & Mínimo & Máximo & Media & Desv. típ. & Varianza \\
\hline Despersonalización & 350 & 0,00 & 13,00 & 3,9714 & 4,01091 & 16,087 \\
\hline $\begin{array}{l}\text { Agotamiento } \\
\text { Emocional }\end{array}$ & 350 & 2,00 & 35,00 & 19,1143 & 9,04814 & 81,869 \\
\hline Realización Personal & 350 & 24,00 & 48,00 & 38,6857 & 6,07682 & 36,928 \\
\hline
\end{tabular}

Fuente: Elaboración propia 
Al analizar las dimensiones obtenidas, es importante examinar cada subescala de manera independiente y no de forma conjunta (Tabla 3), así dimensiones altas en despersonalización y agotamiento emocional y bajas en la realización personal son aspectos que definen el estrés laboral o Síndrome de Burnout. Por ende, los datos que muestra la tabla 3, revelan desigualdad y niveles bajos de despersonalización $(3,97)$ y agotamiento emocional $(19,11)$ mientras que la baja realización personal se mantiene en niveles medios $(38,68)$.

Al realizar el análisis de la relación existente entre la edad y las dimensiones de Burnout (Tabla 4) no se observaron diferencias significativas ya que se reportan valores de significancia bilateral para el Chi cuadrado de Pearson superior a 0.05 , dicho resultado afirma la hipótesis nula, es decir la que no existe ninguna relación entre las dimensiones del Burnout y la edad de los docentes.

\section{Tabla 4}

Relación entre la edad y las dimensiones del Burnout

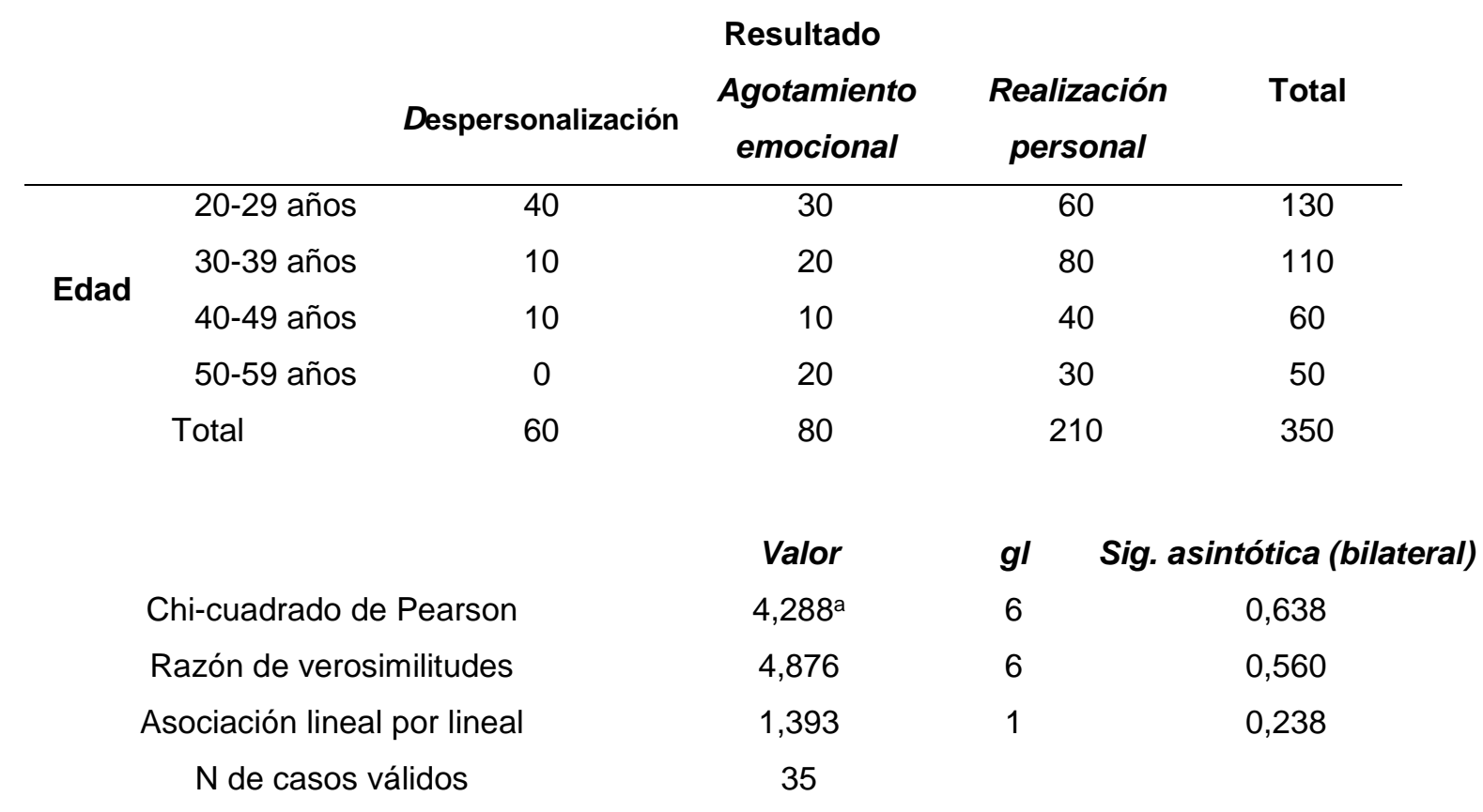

a. 10 casillas $(83,3 \%)$ tienen una frecuencia esperada inferior a 5 . La frecuencia mínima esperada es, 86 .

Fuente: Elaboración propia. 


\section{Tabla 5}

Relación entre la experiencia laboral y las dimensiones del Burnout.

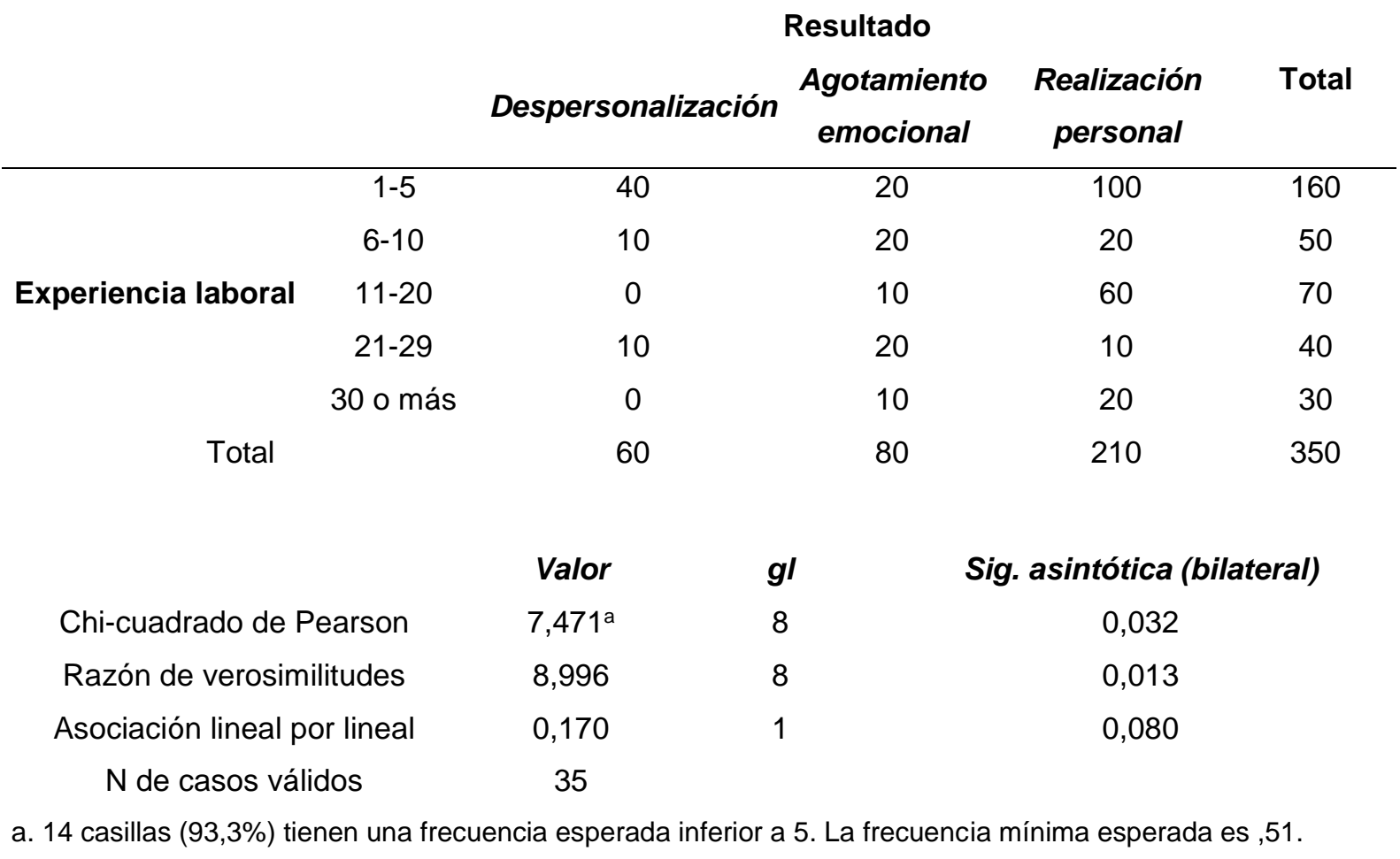

Fuente: Elaboración propia.

La Tabla 5 muestra la existencia de una relación entre la experiencia laboral y las dimensiones de Burnout, debido a que el valor del Chi cuadrado de Pearson es inferior a 0.05 , asumiendo la hipótesis afirmativa como valedera.

Otro resultado importante gira en torno al estrés laboral que se genera de acuerdo al género la Figura 1, muestra que los hombres tienden a poseer un mayor agotamiento emocional con respecto a las mujeres; en cuanto a la despersonalización el género masculino muestra mayores índices con respecto al género femenino y finalmente las damas son aquellas que evidencian mayores índices de realización personal con respecto a los caballeros. 
Femenino

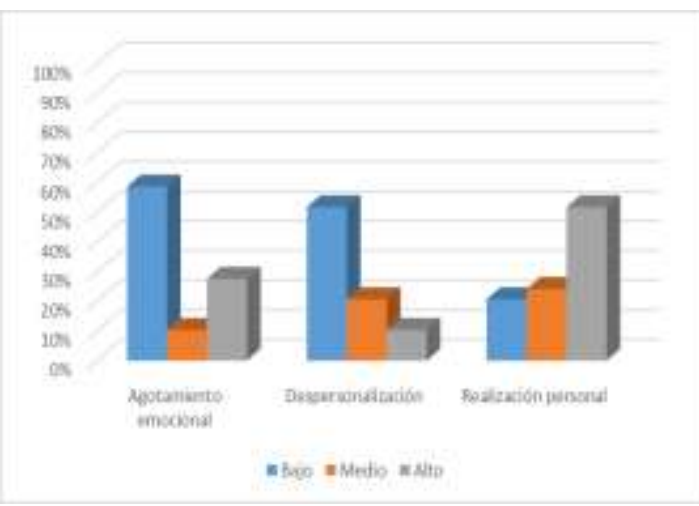

Masculino

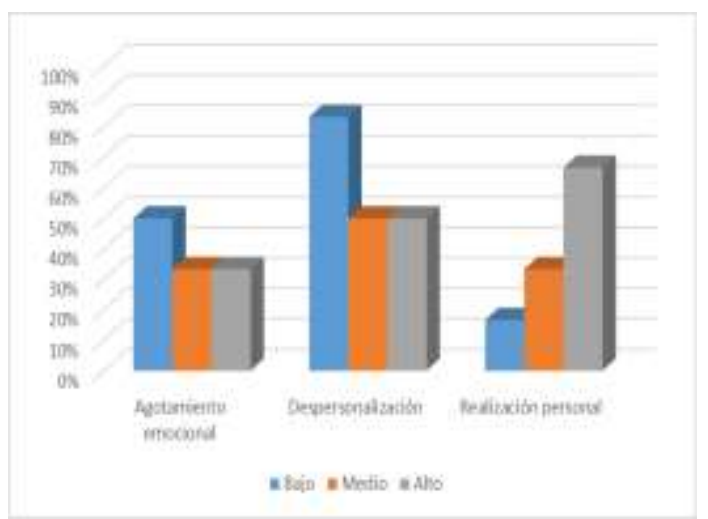

Figura 1. Dimensiones de Burnout por género. Fuente: Elaboración propia

Todos estos resultados dan luces y propician posibles discusiones científicas en torno a la temática, mismas que se detallan a continuación.

\section{DISCUSIÓN}

Los resultados encontrados indican que el nivel de estrés crónico o severo es bajo, para la muestra en mención, pues sólo la tercera parte se ubica en las categorías altas del Síndrome de Burnout, resultado que coincide con el estudio realizado por (Arias, Sánchez, \& Ceballos, 2017), mismo que evidencia la no relación entre los niveles de estrés y la edad de los participantes en el estudio.

En el mismo sentido en cuanto a la variable años de experiencia (Bedoya, Vega, Severiche, \& Meza, 2017) y (Montalvão, Cortez, \& Grossi-Milani, 2018), manifiestan que los docentes más jóvenes presentan mayores niveles de agotamiento laboral que aquellos que poseen mayor edad, además se menciona como vulnerables a aquellos que poseen una experiencia laboral inferior a cinco años. Por el contrario la investigación realizada por (Rivera, Segarra, \& Valverde, 2018), (Salgado \& Leira, 2018), demostró que aquellos docentes que se encuentran en práctica profesional más de 10 años tienen mayores posibilidades de presentar estrés crónico y por tanto Síndrome de Burnout (Burgos-Briones, Cevallos-Ponce \& Anchundia-Párraga, 2019). Un dato que llama fuertemente la atención es que más de la mitad de los encuestados posee índices altos en cuanto a la realización personal, datos similares al estudio realizado por (Bedoya, Vega, Severiche, \& Meza, 2017), este hecho marca de manera 
significativa la buena relación laboral y el ambiente amigable que existe en el trabajo. De acuerdo con (Bustamante, Bustamante, González, \& Bustamante, 2016) y (Arias, Sánchez, \& Ceballos, 2017), las instituciones educativas que buscan estrategias como el coaching, la inteligencia emocional o la gestión adecuada del conocimiento y competencias docentes fortalecen esta dimensión y minimizan la carga del docente y por ende el estrés en el trabajo (Carlotto \& Câmara, 2017).

De acuerdo con (Marenco \& Ávila, 2016), el Síndrome de Burnout posee un carácter multifactorial y por tanto sería conveniente no sólo analizar su espacio de trabajo sino también su entorno personal, ya que el apoyo recibido por familiares y amigos puede disminuir de manera significativa los efectos del cansancio y estrés crónico incidiendo positivamente en su salud y bienestar físico y emocional (Anzules-Guerra, et al., 2019).

\section{PROPUESTA}

Como se ha analizado en la presente investigación, la profesión docente es una de las más vulnerables en relación a poseer niveles elevados de estrés crónico o Síndrome de Burnout, razón de más para precautelar el bienestar integral de los docentes. En base a esta afirmación se hace necesario la intervención de directivos y autoridades de las instituciones educativas, quienes deberían implementar y proponer alternativas que reduzcan o favorezcan ambientes laborales saludables.

Desde ese punto de vista, se plantea la mediación de una propuesta que va dirigida a las dos dimensiones del Burnout en las que se observaron relaciones negativas, por un lado la Despersonalización y por otro el Agotamiento Emocional, la propuesta está dirigida desde dos aspectos, el primero tratando de centrarse en la parte física del docente y la segunda en su parte psicológica, para ello entonces se ofrecen dos herramientas que podrían disminuir las afectaciones de estrés diario al que están expuestos los docentes. 


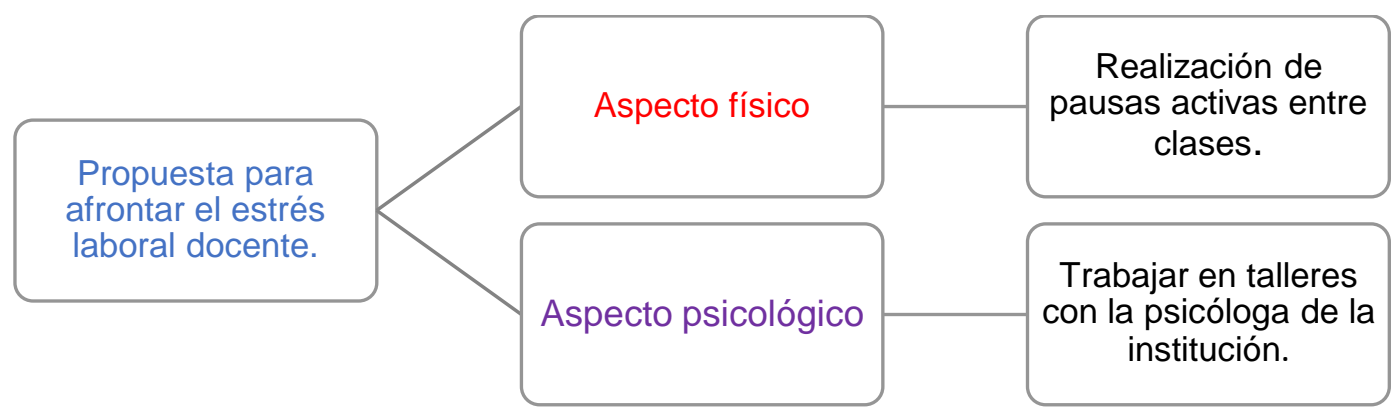

Figura 2. Propuesta para afrontar el estrés laboral docente. Fuente: Elaboración propia

\section{Aspecto Físico}

En párrafos anteriores se exponen algunos de los síntomas que se producen como consecuencia del estrés a nivel físico, para ello se propone la práctica de pausas activas, muchos docentes a lo largo de su jornada laboral tienen breves espacios libres, es ahí donde éste debería aprovechar unos minutos para relajarse y disminuir la tensión muscular. Para la realización de las pausas activas entre clases es importante que tanto docentes como directivos comprendan la necesidad que implica este espacio, para hacer uso de esta pausa activa se plantea que el docente pueda realizarlo en algún espacio de la institución y a ser posible sin interrupción de estudiantes, compañeros $o$ autoridades.

El docente puede escoger entre caminar de manera suave y relajada o al mismo tiempo realizar algún ejercicio de estiramiento que contribuya a disminuir su tensión muscular y el estrés de los días laborables, es muy importante recalcar que el momento de esta pausa activa, lo realizará el docente a nivel individual sin instrucción o guía de otro compañero, la intencionalidad de este evento es que crear un espacio único de privacidad y encuentro consigo mismo. La rutina de las pausas activas debería:

1. Ser realizada durante un mínimo de 5 y un máximo de 10 minutos.

2. Si es posible realizarla dos veces al día.

3. Durante todos los días o por lo menos tres veces a la semana.

4. Ubicarse en un lugar sosegado donde pueda tener privacidad. 
5. Caminar de forma tranquila controlando su respiración o realizando suaves estiramientos.

La idea de proponer pausas activas no debe ser entendida como una pérdida de tiempo, sino más bien como un descanso del trabajo y una inversión en tiempo de calidad que favorecería al docente y al estudiante.

\section{Aspecto Psicológico}

Para trabajar dicho aspecto se propone la mediación del DECE (Departamento de Consejería Estudiantil), en este aspecto el docente tendría del apoyo de sus compañeros y la guía de la psicóloga de la institución, para ello la propuesta se encamina a realizar una vez al mes talleres con los docentes, la idea es conocer cómo afrontar las dificultades o problemas que se hayan suscitado dentro de la práctica laboral.

Al igual que un estudiante es remitido al DECE cuando tiene dificultades, el uso de estos talleres puede ser una alternativa que permita al docente conocerse a sí mismo, aprovechar su tiempo al máximo y minorar la carga excesiva que supone su profesión, la creación de talleres ofrece la oportunidad de trabajar en la parte emocional del docente fomentando el apoyo social entre compañeros.

Esta propuesta tiene el objetivo de enseñar al docente a gestionar sus emociones y tomar el control de sus problemas o dificultades, la gestión del psicólogo debe ser en todo momento asertiva permitiendo al docente dejar aflorar posibles situaciones que están dificultando su desempeño tanto laboral como personal. Para la realización de talleres se debería:

1. Convocar a los docentes una vez terminada la jornada laboral, una vez al mes.

2. Solicitar que puedan llevar ropa cómoda y zapatos deportivos.

3. La duración puede variar entre una hora u hora y media.

4. Permitir que los docentes puedan expresar sus fortalezas y debilidades.

5. Abordar situaciones que hayan ocurrido y brindar apoyo entre todos.

6. Fomentar una escucha y diálogo asertivo. 
7. Finalizar con alguna anécdota divertida o con la posibilidad de compartir algún postre.

Finalmente convendría realizar un seguimiento de las propuestas implementadas, pues una evaluación periódica permitiría retroalimentar si es conveniente continuar con dichas actividades o es necesario mejorar las propuestas de manera que siempre sean vistas como una inversión positiva en pro de la salud del docente y por ende de su desempeño como profesional de la educación.

\section{CONCLUSIONES}

El Síndrome del Quemado o Burnout es un trastorno que afecta en gran medida a personas que prestan sus servicios a otros, siendo la profesión docente una de las más vulnerables. Numerosos estudios coinciden en afirmar la prevalencia de estrés crónico en profesionales de la educación, dicha patología debe ser analizada de manera urgente para prevenir impactos negativos en su salud y su desempeño profesional.

El estrés es considerado como una respuesta adaptativa al medio, sin embargo, si esta perdura en el tiempo ocasiona el Síndrome del quemado o Burnout. Dicha situación afectaría de manera significativa tanto la parte física como emocional de la persona, se caracteriza por afectar a tres dimensiones: Agotamiento emocional, Despersonalización y baja Realización en el trabajo.

Al analizar el contexto de América Latina y más concretamente de Ecuador se puede evidenciar la existencia de varios indicadores que propician el estrés de los docentes: bajos salarios, falta de reconocimiento, inestabilidad laboral (despidos), entre otros suponen factores de riesgo que puede desencadenar en un agotamiento emocional constante de ahí la invitación a que directivos y personal de talento humano busquen generar modulares positivos que actúen a favor de evitar situaciones prolongadas que deriven en estrés a largo plazo.

Para finalizar cabe mencionar, que las instituciones educativas, autoridades y directivos, deberían ahondar en las causas del estrés laboral en sus trabajadores y generar una consciencia que permita establecer políticas o medidas de prevención que sensibilicen esta realidad en el accionar diario de su práctica. Finalmente, el 
individualismo y la competencia deben dejar paso a la solidaridad y cooperación, se trata de un aprendizaje rico en inteligencia emocional y no tanto intelectual, de esta manera el docente podría ejercer con mayor dignidad una profesión muy poco valorada.

\section{FINANCIAMIENTO}

No monetario.

\section{AGRADECIMIENTO}

A los docentes de Educación Básica de las diferentes Unidades Educativas de la ciudad de Cuenca-Ecuador por apoyar la investigación.

\section{REFERENCIAS CONSULTADAS}

Arias, W., Huamani, J., \& Ceballos, K. (2019). Burnout Syndrome in School teachers and University Professors: A Psychometrical and Comparative Analysis from Arequipa City. Propósitos y Representaciones, 7(3), 72-110. doi:http://dx.doi.org/10.20511/pyr2019.v7n3.390

Arias, W., Sánchez, M., \& Ceballos, K. (2017). Síndrome de Burnout, satisfacción laboral y relaciones interpersonales en profesores de una institución educativa de Arequipa [Burnout syndrome, job satisfaction and interpersonal relationships in teachers of an educational institution in Arequipa]. Acta Psicológica Peruana 2 (1), 118- 139. Obtenido de https://n9.cl/rrf1m

Anzules-Guerra, J., Véliz-Zevallos, I., Vinces-Centeno, M., \& Menéndez-Pin, T. (2019). Síndrome de burnout: Un riesgo psicosocial en la atención médica. Revista Arbitrada Interdisciplinaria de Ciencias de la Salud. Salud y Vida, 3(6), 839-859. http://dx.doi.org/10.35381/s.v.v3i6.393

Bambula, F., \& Gómez, I. (2016). La investigación sobre el síndrome de burnout en latinoamérica entre 2000 y el 2010 [Research on burnout syndrome in Latin America between 2000 and 2010]. Psicología desde el Caribe, 33(1), 114- 132. doi:http://dx.doi.org/10.14482/psdc.33.1.8065 
Bedoya, E., Vega, N., Severiche, C., \& Meza, M. (2017). Síndrome de Quemado (Burnout) en Docentes Universitarios: El Caso de un Centro de Estudios del Caribe Colombiano [Burnout Syndrome in University Teachers: The Case of a Colombian Caribbean Studies Center]. Formación Universitaria, 10(6), 51- 58. Obtenido de https://n9.cl/xgad

Bustamante, E., Bustamante, F., González, G., \& Bustamante, L. (2016). El burnout en la profesión docente: un estudio en la escuela de bioanálisis de la Universidad de Carabobo Sede Aragua, Venezuela [Burnout in the teaching profession: a study in the bioanalysis school of the University of Carabobo Sede Aragua, Venezuela]. Scielo, 62, 111- 121. Obtenido de http://scielo.isciii.es/pdf/mesetra/v62n243/original2.pdf

Burgos-Briones, J., Cevallos-Ponce, V., \& Anchundia-Párraga, J. (2019). Síndrome de Burnout y calidad educativa docente. Revista Arbitrada Interdisciplinaria Koinonía, 4(8), 739-751. http://dx.doi.org/10.35381/r.k.v4i8.481

Carlotto, M., \& Câmara, S. (2017). Riscos psicossociais associados à síndrome de burnout em professores universitários C. Avances en Psicología Latina, 35(3), 447-

457. doi:http://dx.doi.org/10.12804/10.12804/revistas.urosario.edu.co/apl/a.4036.

Contreras, M., \& Burguillos, A. (2014). Estrés y Burnout en profesores de primaria y secundaria de Huelva: Las estrategias de afrontamiento como factor de protección [Stress and Burnout in primary and secondary teachers in Huelva: Coping strategies as a protection factor]. INFAD Revista de Psicología, №1Vol.4, 303-310. Obtenido de https://n9.cl/zyyf

Esteras, J., Chorot, P., \& Sandín, B. (2019). Sintomatología física y mental asociada al síndrome de burnout en los profesionales de la enseñanza [Physical and mental symptoms associated with burnout syndrome in teaching professionals]. Revista de Psicopatología y Psicología Clínica(24), 29-37. Obtenido de https://n9.cl/ylgp

Gallegos, W., \& Barrios, N. (Marzo de 2013). Síndrome de burnout en docentes de Educación Básica Regular de Arequipa [Burnout syndrome in teachers of Regular Basic Education of Arequipa]. Educación Vol. XXII, 53-76. Obtenido de https://n9.cl/ahf9

González, N. (2008). Prevalencia del Estrés en la satisfacción laboral de los docentes universitarios [Prevalence of Stress in the job satisfaction of university teachers]. REDHECS: № 4 Revista electrónica de Humanidades, Educación y Comunicación Social, 68-89. Obtenido de https://n9.cl/qhm8 
Marenco, A., \& Ávila, J. (2016). Dimensiones de apoyo social asociadas con Síndrome de Burnout en docdentes de media académica [Dimensions of social support associated with Burnout Syndrome in academic average students]. Pensamiento Psicológico, 14(2), 7-18. Obtenido de https://n9.cl/ypp5

Montalvão, C., Cortez, L., \& Grossi-Milani, R. (2018). Síndrome de Burnout e condições psicossociais em docentes do ensino superior. Acta Scientiarum. Human and Social Sciences, 40(3). doi:https://doi.org/10.4025/actascihumansoc.v40i3.36437

Quintero, J., Vergara, P., Hernández, R., Osorio, F., \& Orozco, E. (2018). Prevalencia del Síndrome de Burnout en docentes: Factores asociados al estatuto de vinculación laboral en Colombia [Prevalence of Burnout Syndrome in teachers: Factors associated with the statute of employment relationship in Colombia]. Revista Interamericana de Psicología Ocupacional, 119-133. Obtenido de https://n9.cl/9nu1

Rabasa, B., Rabasa, B. F.-F., Gil-Monte, P., \& Llorca-Pellicer, M. (2016). El papel de la culpa en la relación entre el síndrome de quemarse por el trabajo y la inclinación al absentismo de profesores de Enseñanza Secundaria [The role of guilt in the relationship between the syndrome of burning from work and the inclination to ]. Revista de Psicodidáctica, 21(1), 103- 119. Obtenido de https://n9.cl/smfvm

Rivera, Á., Segarra, P., \& Valverde, G. (2018). Síndrome de Burnout en docentes de instituciones de educación superior [Burnout syndrome in teachers of higher education institutions]. Archivos Venezolanos de Farmacología y Terapéutica, 37(2), 17-23. Obtenido de https://n9.cl/qkrd

Romero, A., Pimienta, I., Ramos, M., Sánchez, A., \& González, R. (2019). Niveles de síndrome de burnout en docentes de una universidad ecuatoriana [Levels of burnout syndrome in teachers of an Ecuadorian university]. Dilemas Contemporáneos: Educación, Política y Valores(3). Obtenido de https://n9.cl/dyxpt

Salgado, J., \& Leira, F. (2018). Síndrome de burnout y calidad de vida profesional percibida según estilos de personalidad en profesores de educación primaria [Burnout syndrome and perceived professional quality of life according to personality styles in primary school teachers]. CES Psicología, 11(1), 69- 89. Obtenido de https://n9.cl/noi9

Silva, M., García, R., González, M., \& Ratto, A. (2015). Prevalencia del Síndrome de quemarse por el trabajo y variables sociodemográficas en un grupo de maestras de Montevideo [Prevalence of Burning Syndrome from work and sociodemographic variables in a group of teachers from Montevideo]. Ciencias Psicológicas, 9(1), 55- 62. Obtenido de https://n9.cl/m5u8n 
Villaverde, D., Unda, S., Escotto, E., \& Flores, R. (2019). Personality Traits that Predict the Burnout Syndrome on. Propósitos y Representaciones, 7(3), 41- 56. doi:http://dx.doi.org/10.20511/pyr2019.v7n3.346.

Vintimilla-Veloz, M. G., Erazo-Álvarez, J. C., \& Narváez-Zurita, C. I. (2020). Branding e identidad corporativa en el sector financiero popular y solidario [Branding and corporate identity in the popular and solidarity financial sector]. Revista Arbitrada Interdisciplinaria Koinonía, 255-286. doi:http://dx.doi.org/10.35381/r.k.v5i10.695

Zavala, J. (2008). Estrés y burnout docente: conceptos, causas y efectos [Teaching stress and burnout: concepts, causes and effects]. Educación, 17(32), 67-86. Obtenido de $h$ ttps://n9.cl/26iy 